\title{
Spectres of Ambiguity in Divergent Thinking and Perceptual Switching
}

\author{
Mihaela Taranu* \\ CogNovo \\ School of Psychology, \\ Plymouth University, UK \\ *Mihaela.Taranu@CogNovo.eu
}

\author{
Frank Loesche \\ CogNovo \\ School of Computing, Electronics and Mathematics, \\ Plymouth University, UK
}

Received 12 May 2017; accepted 30 September 2017; published 21 November 2017.

\begin{abstract}
Divergent thinking as a creative ability and perceptual switching between different interpretations of an unchanging stimulus (known as perceptual multistability) are thought to rely on similar processes. In the current study, we investigate to what extent task instructions and inherent stimulus characteristics influence participants' responses. In the first experiment, participants were asked to give as many interpretations for six images as possible. In the second experiment, participants reported which of two possible interpretations they saw at any moment for the same line drawings. From these two experiments, we extracted measures that allow us direct comparison between tasks. Results show that instructions have a large influence over the perception of images traditionally used in two different paradigms and that these images can be perceived in appropriate ways for both tasks. In addition, we suggest that the connection between the two phenomena can be explored interchangeably through three experimental manipulations: a) using a common set of images across both experiments, b) giving different task instructions for the two tasks, and c) extracting comparable metrics from both experimental paradigms.
\end{abstract}

Keywords: ambiguity; divergent thinking; perceptual switching. 


\section{Introduction}

Every day people face situations for which new ways of seeing and thinking are necessary or at least beneficial. How are they able to arrive at these novel interpretations? Here we explore two approaches to answer this question: investigating creative problem solving (novel ways of reaching a goal) and perceptual switching (different ways of interpreting the environment). We also investigate their connection through homogeneous measurements.

Creative problem solving refers to the process of generating original and appropriate responses to reach a goal. The ability of divergent production (the generation of many possible solutions), which is instrumental to creative problem solving (see Guilford, 1967), can be assessed in numerous ways (Runco \& Pritzker, 2011, p. 548). Generally, in divergent thinking tasks people are instructed to generate as many ideas as possible for visual or verbal stimuli. The number of generated distinct ideas provides a measurement of fluency or flexibility and is thought to reflect the ability to mentally restructure the stimulus.

On the other hand, consider ambiguous images such as Jastrow's bistable duckrabbit (Jastrow, 1900, p. 295): people first perceive one of the interpretations, then after prolonged viewing the second interpretation is perceived, after which perception alternates between the two interpretations of "duck" and "rabbit" (Leopold \& Logothetis, 1999; Long \& Toppino, 2004). The phenomenon of switching between alternative interpretations of ambiguous images is generally known as multistable perception, or as bistable perception if only two interpretations are possible (Leopold \& Logothetis, 1999). Multistability is considered to involve restructuring the interpretations of the ambiguous stimulus at higher levels of cognitive processing (Long \& Toppino, 2004; Sterzer \& Kleinschmidt, 2007).

Both divergent thinking and perceptual switching tasks attempt to measure the influence of perceptual and mental restructuring and are therefore thought to rely on similar processes (Schooler \& Melcher, 1995). Phenomenologically, switching between different representations in divergent thinking tasks and different interpretations of an ambiguous image have been described as similar human experiences since the early $20^{\text {th }}$ century by Gestalt theories (Duncker, 1963). Researchers interested in creative problem solving and multistability have been both hunting for and haunted by ambiguity for a long time. However, the commonalities between creative problem solving and perceptual switching have predominantly been observed anecdotally. Only a few studies have empirically investigated the relationship between the two phenomena.

Wiseman, Watt, Gilhooly, and Georgiou (2011) provided empirical support for the connection between perceptual switching and divergent thinking or, in general, creative ability: participants who reported that they could switch more easily to the second interpretation of the Jastrow duck-rabbit image also rated themselves as being 
more artistically creative and better creative problem solvers. They also found a strong correlation between self-reported ease of perceptual switching and categorical flexibility measured with a single item unusual uses task. Following up on these results, Doherty and Mair (2012) found that fluency for written responses measured with the Pattern Meanings task (Wallach \& Kogan, 1965, p. 33) is positively correlated with the number of switches in perceptual multistability tasks (duck-rabbit, vase-face, Necker cube). The authors speculated that the same executive control mechanisms may be involved in perceptual switching and divergent production, therefore the relationship is worth exploring further.

The effect of ambiguous visual stimuli on divergent thinking was also investigated by $\mathrm{Wu}, \mathrm{Gu}$, and Zhang (2016) and Laukkonen and Tangen (2017). In both studies, ambiguous and non-ambiguous images were presented before a creativity task (i.e., an alternative uses task or insight problem, respectively). The results of both studies showed that participants could generate a significantly higher number of solutions in the creativity task if they saw an ambiguous figure instead of an unambiguous image. This was interpreted as evidence that ambiguous images facilitate creative ideas, possibly due to the fact that both involve resolving conflicting sensory input.

The three studies by Wiseman et al. (2011), Wu et al. (2016), and Laukkonen and Tangen (2017) show that performance in creative problem solving and perceptual switching tasks are related and can even influence each other. However, it is hard to accommodate results across tasks and studies that use different measures of divergent thinking and multistable perception.

In this study, we took images regularly used in divergent thinking and bistable perception tasks and explored whether the way they were administered affected participants' performance. Specifically, we investigated whether the images from the two paradigms led to similar temporal measures when administered in divergent thinking and perceptual switching conditions. To explore this hypothesis, we used images from a divergent thinking task, namely the Pattern Meaning task by Wallach and Kogan (1965), and compared participants' task performance with ambiguous visual stimuli such as duckrabbit, mouse-man, donkey-seal, which are widely used in bistable research (see Wimmer, Doherty, \& Collins, 2011). Finally, additional temporal measurements were extracted from the computerized divergent thinking and bistable perception tasks.

With this study, we aim to investigate to what extent task instructions and inherent stimulus characteristics influence participants' responses. Specifically, we ask whether the connection between the two phenomena can be investigated interchangeably by observing three experimental manipulations: a) using a shared set of images in both tasks, b) giving different task instructions, and c) extracting comparable metrics from both experimental paradigms. We also aim to contribute to the growing literature of empirical investigations of the relationship between divergent thinking and multistable perception. 
In the first experiment, we explored whether images taken from bistable perception tasks, which are typically considered to have two interpretations, can trigger more interpretations, similarly to the images used in the divergent thinking tasks. Therefore, we presented images from divergent thinking and perceptual switching tasks to a group of participants and asked them to generate as many interpretations as possible. This is a typical administration of a divergent thinking task in creativity research, for example in Wallach and Kogan (1965).

In the second experiment, participants were presented with the same set of images in a perceptual switching task. In this case, participants were asked for an initial interpretation of one image, then shown two possible interpretations and later asked to continuously report their perception for 120 seconds. This is a setup typically employed in bistability experiments. We examined whether perceptual switching dynamics differed depending on the origin of the images.

In addition, we explored ways of directly comparing results from the two experiments. Besides the measurements which are normally used, we recorded the time participants took to generate the first answer in the divergent thinking experiment. This is similar to the concept of an initial reaction time, which is often used in the analysis of perceptual switching tasks, i.e., the time taken to report the first interpretation by button press. An additional measurement was the first phase duration, which denotes the time it took participants to name their first solution in the divergent thinking task and the time until they gave the first interpretation in the perceptual switching task. We were interested to see to what extent these two variables were similar for the same image between experiments.

\section{Experiment 1: Divergent Thinking}

\section{Participants}

Six postgraduate students aged between 25 and 48 years (mean $=37.17, \mathrm{SD}=8.06$ ) participated (self-reported gender: 1 female, 3 males, 2 unspecified).

\section{Materials and Procedure}

Six different images were used, three of them taken from the Pattern Meaning task by Wallach and Kogan (1965), and three frequently used in bistable perception tasks (see Figure 1). 


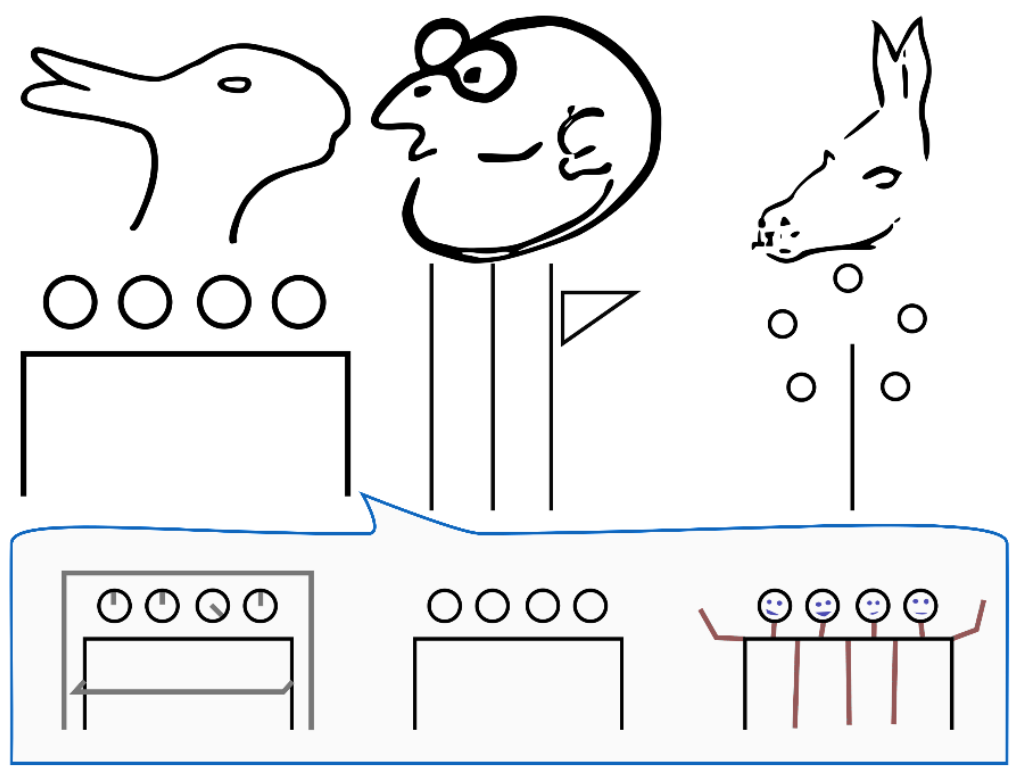

Figure 1. Images used in the two experiments; top row: duck-rabbit, manmouse, seal-donkey; center row: oven-people, street map-flag pole, sparkling magic wand-flower; bottom row: disambiguated image for oven, ambiguous oven-people, disambiguated image for group of people.

The setup of the computerized task for each image consisted of the following three stages: "instruction," "task," and "break." During the "instruction" stage, participants were told that they should give "as many answers as [they] can" for each of the stimuli and that there were no correct or incorrect answers. Each of the six images was presented for 120 seconds, during which time participants reported their interpretations. This was followed by a self-paced "break."

For this exploratory study, fluency was chosen as one of the main measures as used in the Pattern Meaning task and other divergent thinking tasks because it can be scored objectively for any sample size. Participants' verbal responses were recorded and later transcribed along with the start and end time of each answer. Fluency was extracted as the number of responses generated during the 120 seconds of the "task," the initial reaction time (the response time $t_{1}$ from the start of the task until a participant gave the first answer) and the first phase duration (the time participants maintained their first interpretation). 


\section{Results}

Fluency as the number of generated ideas is summarized in Figure 2. All participants were able to produce two or more distinct interpretations for each stimulus. The average fluency per participant across all six tasks was between 4.17 and 8.33 and the initial answer was produced between $1.67<t_{1}<8.46$ seconds after task start. The average fluency for images from divergent thinking tasks across all participants (mean $=6.50, \mathrm{SD}=2.31$ ) was very similar to the average for images from perceptual switching tasks (mean $=6.44, \mathrm{SD}=3.17$ ).

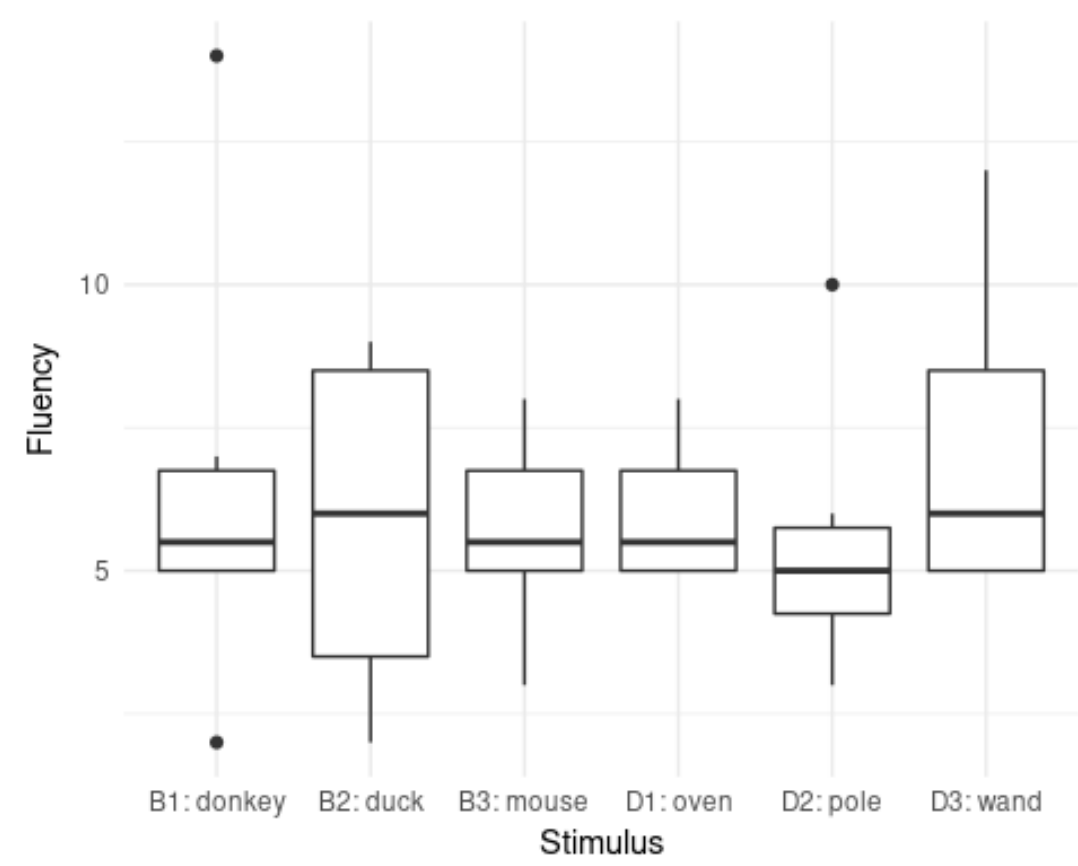

Figure 2. Number of solutions for each stimulus. The six stimuli are displayed on the $x$-axis and the number of solutions is displayed on the $y$-axis. Each of the boxes span from the first to the third quartile of the distribution of fluency; the thick line represents the median.

\section{Experiment 2: Perceptual Switching}

\section{Participants}

Six participants (five females, one male), aged between 27 and 33 years (mean $=30.17$, $\mathrm{SD}=2.32$ ) participated.

\section{Materials and Procedure}

The same six images used in Experiment 1 were presented in a computerized perceptual switching condition (see Figure 1). A sequence of stages was administered for each image: "initial interpretation," "disambiguation," "training," "task," and "break." 
Initially, participants were asked to write down their "initial interpretation" of the image. To disambiguate each line drawing from the Pattern Meaning task, the two most frequent interpretations from another study were visualized (see Figure 1). During the "disambiguation," these were used to instruct participants which interpretations of the images they should report. For example, participants who saw the image at the bottom center of Figure 1 were instructed to switch between the "oven" (bottom left) and "people" (bottom right) interpretation. In the self-paced "training," pressing one of the two defined keys on the computer keyboard showed the corresponding disambiguation on screen. During the 120 seconds of the "task," the states of the two keys were recorded continuously. The start and end times for each button press served as the basis to calculate variables such as phase duration (the length of time during which one interpretation is sustained), first phase durations and initial reaction times. At the end of each sequence of stages, participants had self-paced "breaks."

\section{Results}

The first phase durations for each stimulus are displayed in Figure 3. The two interpretations were generally well balanced (Moreno-Bote, Shpiro, Rinzel, \& Rubin, 2010) for all six images, suggesting that participants perceived equally the two interpretations for each image. This shows that perceptual switching can be experienced in response to images taken from divergent thinking tasks if participants are instructed to do so.

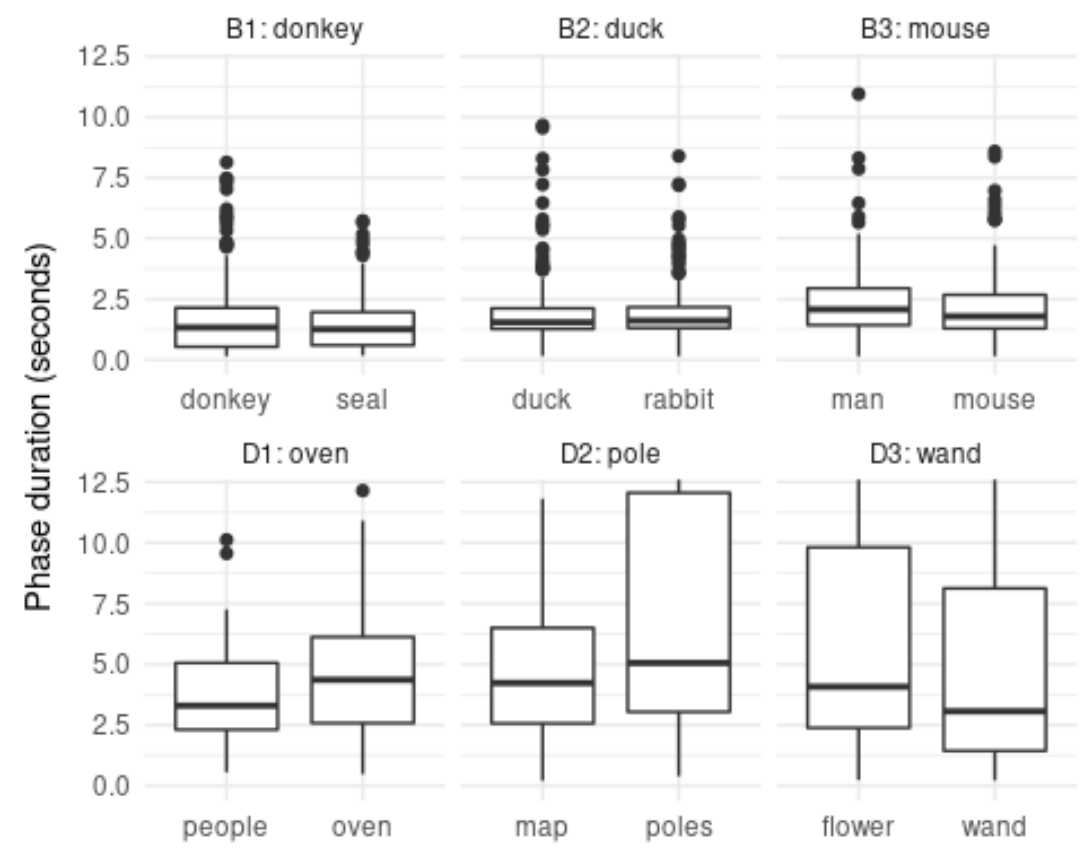

Figure 3. The average phase duration for each interpretation of each stimulus. The phase duration (in seconds) is displayed on the $y$-axis, while the interpretations of the stimuli are displayed on the $x$-axis. 
In addition to the separate analysis of the two experiments, we explored a direct experimental connection between divergent thinking and perceptual switching. For example, we extracted the initial reaction time and the first phase durations for each image from both experiments.

The initial reaction times of all participants for each of the six images is shown separately for the two experiments in Figure 4. Comparing the same image across the two experiments seems to indicate that the instructions influenced the time it took participants to generate an answer. Specifically, the initial reaction time in the divergent thinking tasks was generally shorter (median $=2.98 \mathrm{~s}$ ) than in the perceptual switching task (median $=3.44 \mathrm{~s}$ ), even though the data collected does not provide enough evidence to suggest a significant difference. Moreover, Figure 4 provides no indication that the initial reaction times for the three images taken from the Pattern Meaning task are different from the images taken from the perceptual switching tasks. Nevertheless, Figure 4 seems to show that some images elicit a longer time until the first interpretation in both experiments (e.g., pole-street), while other images (e.g., oven-group of people) cause shorter reaction times in both experiments.

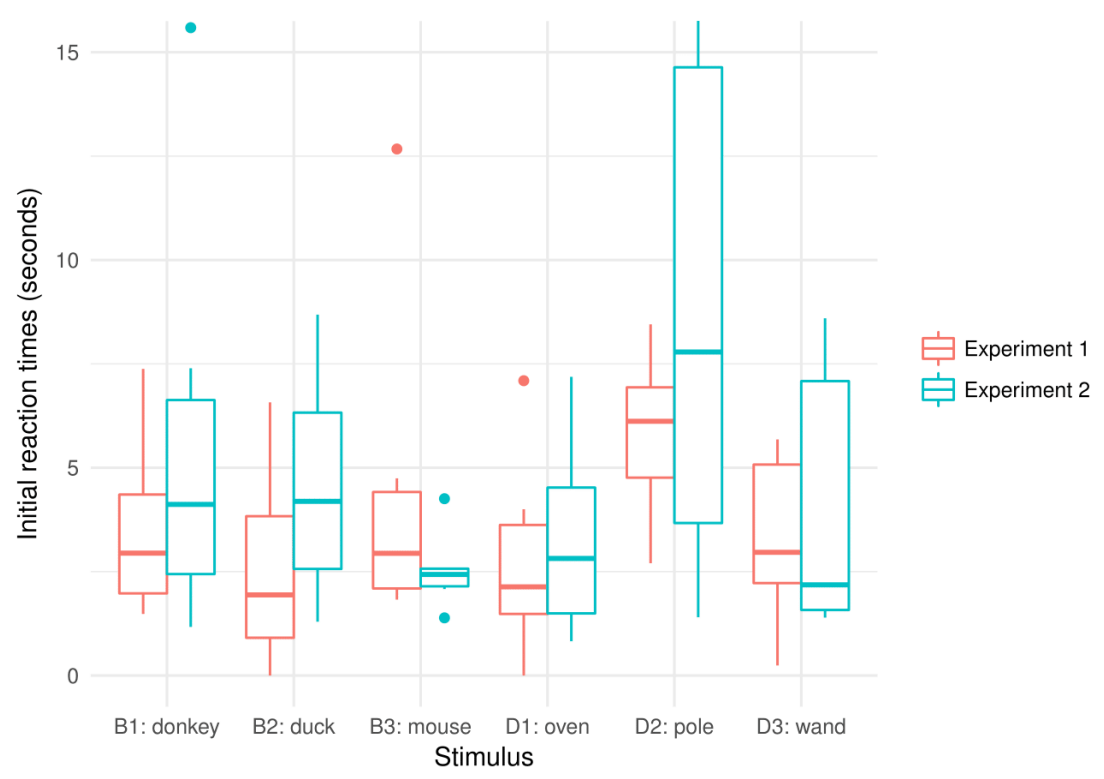

Figure 4. Distributions of the initial reaction times for each of the six images, separately for the two experiments. 
Figure 5 displays the first phase duration for each image across all participants and experiments. It also seems to indicate that the first phase duration for some images is short for both experiments (e.g., donkey-seal and duck-rabbit) while participants maintain the first interpretation for a longer time for line drawings (e.g., street mapflag pole). Overall, the first phase duration for the divergent thinking task in Experiment 1 is shorter (median $=1.71 \mathrm{~s}$ ) than for the perceptual switching task in Experiment 2 (median $=2.52 \mathrm{~s})$.

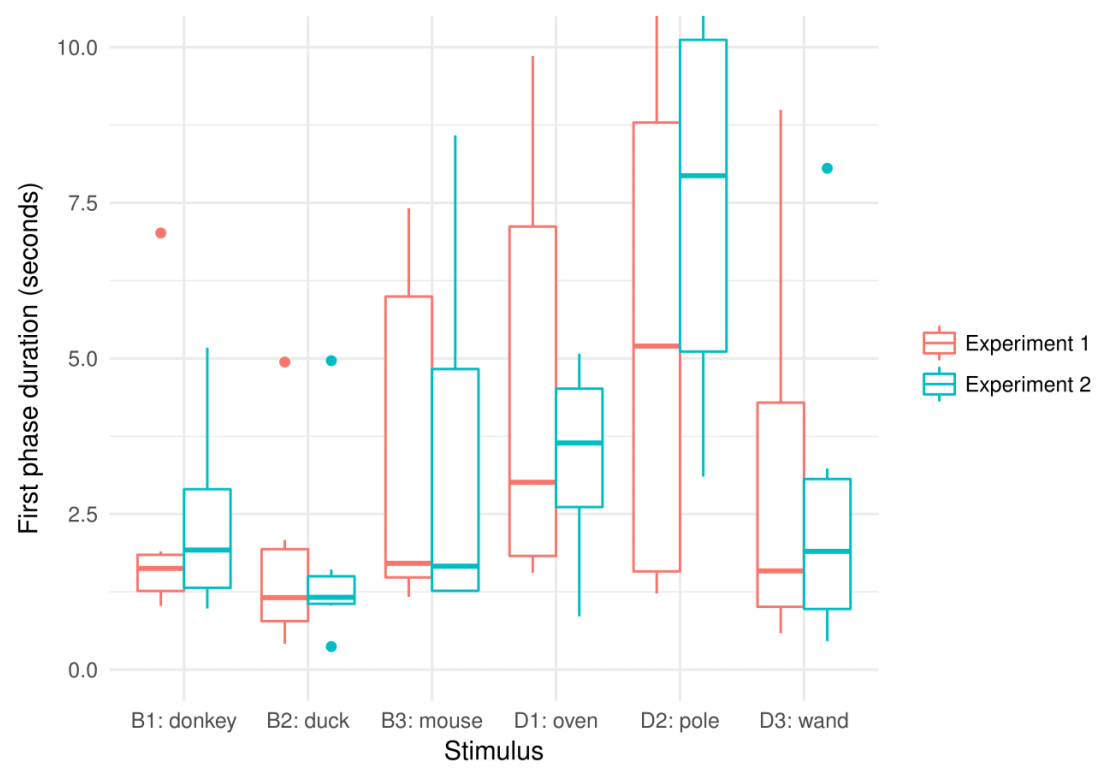

Figure 5. Distribution of the first phase duration for each of the six images, separately for the two experiments.

\section{Discussion}

The aim of the current study was to explore the connection between divergent thinking and perceptual switching. To investigate this, we collected a set of images previously used in divergent thinking or perceptual switching tasks. We then observed to what extent this combined set of images can be used in each of the original tasks. Moreover, we aimed to investigate whether participants' responses could indicate whether the original source of the image had an impact on the results. This would allow us to identify whether, except for the instructions, there are inherent characteristics that differentiate the two sources of images.

In the first experiment, images taken from divergent thinking and perceptual switching paradigms were presented to participants as typically done in divergent thinking tasks, while in the second experiment the same images were presented as typically done in perceptual switching tasks. The influence of instructions in the two tasks can 
be observed, for example, when we compare the initial reaction times and first phase durations for the same image from the two experiments. For both measures the results showed that instructions lead to different results based on the two tasks that participants were instructed to complete. These results indicate that instructions play a significant role in the way different images are interpreted, a claim that is supported by previous research on the influence of instructions on divergent production (Runco, Illies, \& Eisenman, 2005). The role of instructions for perceptual switching needs to be addressed more thoroughly by future research.

In Experiment 1, we found that the number of solutions does not substantially differ depending on the source of the image. This suggests that images previously used in perceptual switching tasks can have more possible interpretations than usually assumed for bistable perception-at least if instructions require this.

The phase durations measured in Experiment 2 do not indicate a clear distinction between the source of the line drawings; i.e., perceptual switching dynamics cannot be used to distinguish between images taken from perceptual switching or divergent thinking tasks. This suggests that bistable perception can also occur to some extent in response to the images taken from the divergent thinking task.

The number of participants in each experiment is small and no statistical inferences can be drawn. Nevertheless, trends indicate that there are individual differences across the selected images, independently of their original source. This result supports findings from bistable research showing that perceptual switching differs across images (see van Ee, van Dam, \& Brouwer, 2005). For example, in Experiment 2 the first phase duration for image D2 (street map-flag pole, see Figure 5) is longer than for other images in the same experiment, suggesting that this image is processed differently. Previous studies that examined the connection between creativity and perceptual switching overlooked the role of different types of images (Doherty \& Mair, 2012; Laukkonen \& Tangen, 2017; Wiseman et al., 2011; Wu et al., 2016). It is possible that the relation between perceptual switching and creativity is found only for some images. We suggest further exploration of the origin of these differences, as they might unveil common factors affecting the relationship between the two phenomena.

One challenge in designing the perceptual switching task for Experiment 2 was to select images that were previously used in divergent thinking tasks and which could be disambiguated. Unlike the disambiguation of images from bistable research which had previously been shown to work, the disambiguation of the line drawings from Wallach and Kogan's Pattern Meaning task had not been used before. We selected the two most common answers from a previously recorded data set for three of the images from the Pattern Meaning task. Subsequently we used them to disambiguate the line drawings by adding lines (for an example, see Figure 1). The disambiguations from perceptual switching tasks, on the other hand, consist of slight changes to the position and shape of key elements of the original image. The current results do not 
allow us to draw any conclusions about whether the interpretations we selected for the disambiguation were the easiest to switch between, or whether other interpretations would have worked better. Feedback provided by some participants suggested that they could differentiate between the two types of images based on the way they were disambiguated. Participants described this difference in terms of difficulty to actively imagine additional features for images taken from the divergent thinking task, while for the images from perceptual switching tasks this was easier.

Further differences between the two sets of images are that the images from the Pattern Meaning task are more abstract and are based only on a few geometric shapes such as circles, lines and squares. On the other hand, the images from the perceptual switching tasks can be described as more complex and organic and are supposed to depict real objects (Strüber \& Stadler, 1999). Different reaction times to abstract line drawings can be explained by the greater difficulty in processing these images as compared to content-based depictions of real-world objects. This highlights the importance of the top-down effects of imagery and memory on the perception of images, as previously summarized in Scocchia, Valsecchi, and Triesch (2014).

Another limitation of the study comes from the inability to control for participants' prior exposure to the images. Bistable stimuli are well-known images that are often referenced in arts and popular culture and participants could have known some of them. Therefore, individual prior exposure to these images might have influenced the responses in both experiments. For instance, most of the participants reported knowing the duck-rabbit image beforehand. This might have biased their responses in the divergent thinking condition, particularly if they attempted to overcome the initial fixation on the two known interpretations. Previous experience with the images might also have shortened the time of the first perceptual switch in Experiment 2. Future studies should seek to eliminate the effect of stimuli familiarity, which can affect participants' responses.

\section{Conclusion}

The present study shows that inherent stimuli characteristics and instructions play an important role in interpreting ambiguous images. Participants respond to images from divergent thinking and perceptual switching tasks according to the instructions. Specifically, participants are able to provide more than two unique solutions when instructed to do so, even for images that are typically considered to have only two interpretations. Similarly, they can be instructed to switch back and forth between two given interpretations for images that are considered to have several possible interpretations. In addition, shared metrics can be extracted from both paradigms which would allow researchers a more direct comparison between the two phenomena. 


\section{Acknowledgements}

We would like to thank Monika Chylińska and Susan L. Denham for the discussions and constructive feedback on improving previous versions of this article.

This work was supported by Plymouth University and CogNovo (FP7-PEOPLE-2013ITN-604764), a project funded by the EU Marie Skłodowska Curie programme.

\section{References}

Doherty, M. J., \& Mair, S. (2012). Creativity, ambiguous figures, and academic preference. Perception, 41(10), 1262-1266. doi:10.1068/p7350

Duncker, K. (1963). Zur psychologie des produktiven denkens (2nd ed.). Berlin, Germany: Springer-Verlag. doi:10.1007/978-3-642-49855-8

Guilford, J. P. (1967). The nature of human intelligence. New York, NY: McGraw-Hill.

Jastrow, J. (1900). Fact and fable in psychology. Boston, MA: Houghton-Mifflin.

Laukkonen, R. E., \& Tangen, J. M. (2017). Can observing a Necker cube make you more insightful? Consciousness and Cognition, 48, 198-211. doi:10.1016/j.concog.2016.11.011

Leopold, D. A., \& Logothetis, N. K. (1999). Multistable phenomena: Changing views in perception. Trends in Cognitive Sciences, 3(7), 254-264. doi:10.1016/s1364-6613(99)01332-7

Long, G. M., \& Toppino, T. C. (2004). Enduring interest in perceptual ambiguity: Alternating views of reversible figures. Psychological Bulletin, 130(5), 748-768. doi:10.1037/00332909.130.5.748

Moreno-Bote, R., Shpiro, A., Rinzel, J., \& Rubin, N. (2010). Alternation rate in perceptual bistability is maximal at and symmetric around equi-dominance. Journal of Vision, 10(11), 1. doi:10.1167/10.11.1

Runco, M. A., Illies, J. J., \& Eisenman, R. (2005). Creativity, originality, and appropriateness: What do explicit instructions tell us about their relationships? The Journal of Creative Behavior, 39(2). doi:10.1002/j.2162-6057.2005.tb01255.x

Runco, M. A., \& Pritzker, S. R. (Eds.). (2011). Encyclopedia of creativity (2nd ed.). Boston, MA: Academic Press.

Schooler, J. W., \& Melcher, J. (1995). The ineffability of insight. In S. M. Smith, T. B. Ward, \& R. A. Finke (Eds.), The creative cognition approach (pp. 97-133). Cambridge, MA: MIT Press.

Scocchia, L., Valsecchi, M., \& Triesch, J. (2014). Top-down influences on ambiguous perception: The role of stable and transient states of the observer. Frontiers in Human Neuroscience, 8. doi:10.3389/fnhum.2014.00979

Sterzer, P., \& Kleinschmidt, A. (2007). A neural basis for inference in perceptual ambiguity. Proceedings of the National Academy of Sciences of the United States of Amercia, 104(1), 323-328. doi:10.1073/pnas.0609006104 
Strüber, D., \& Stadler, M. (1999). Differences in top-down influences on the reversal rate of different categories of reversible figures. Perception, 28(10), 1185-1196. doi:10.1068/p2973

van Ee, R., van Dam, L., \& Brouwer, G. (2005). Voluntary control and the dynamics of perceptual bi-stability. Vision Research, 45(1), 41-55. doi:10.1016/j.visres.2004.07.030

Wallach, M. A., \& Kogan, N. (1965). Modes of thinking in young children: A study of the creativityintelligence distinction. New York, NY: Holt, Rinehart \& Winston.

Wimmer, M. C., Doherty, M. J., \& Collins, W. A. (2011). The development of ambiguous figure perception. Monographs of the Society for Research in Child Development, 76(1), i-130.

Wiseman, R., Watt, C., Gilhooly, K., \& Georgiou, G. (2011). Creativity and ease of ambiguous figural reversal. British Journal of Psychology, 102(3), 615-622. doi:10.1111/j.20448295.2011.02031.x

Wu, X., Gu, X., \& Zhang, H. (2016). The facilitative effects of ambiguous figures on creative solution. The Journal of Creative Behavior. doi:10.1002/jocb.161 